Mathematical Modelling and Analysis

Volume 19 Number 5, November 2014, 627-646

http://dx.doi.org/10.3846/13926292.2014.979453

(c) Vilnius Gediminas Technical University, 2014
Publisher: Taylor\&Francis and VGTU

http://www.tandfonline.com/TMMA

Print ISSN: 1392-6292

Online ISSN: 1648-3510

\title{
Effective Numerical Algorithm for Simulations of Beam Stabilization in Broad Area Semiconductor Lasers and Amplifiers
}

\author{
Mindaugas Radziunas ${ }^{a}$ and Raimondas Čiegis ${ }^{b}$ \\ ${ }^{a}$ Weierstrass Institute \\ Mohrenstrasse 39, 10117 Berlin, Germany \\ ${ }^{b}$ Vilnius Gediminas Technical University \\ Sauletekio al. 11, LT-10223 Vilnius, Lithuania \\ E-mail(corresp.): radziuna@wias-berlin.de \\ E-mail: rc@vgtu. It
}

Received May 21, 2014; revised October 10, 2014; published online November 1, 2014

\begin{abstract}
A $2+1$ dimensional PDE traveling wave model describing spatial-lateral dynamics of edge-emitting broad area semiconductor devices is considered. A numerical scheme based on a split-step Fourier method is presented. The domain decomposition method is used to parallelize the sequential algorithm. The parallel algorithm is implemented by using Message Passing Interface system, results of computational experiments are presented and the scalability of the algorithm is analyzed. Simulations of the model equations are used for optimizing of existing devices with respect to the emitted beam quality, as well as for creating and testing of novel device design concepts.
\end{abstract}

Keywords: broad area device, traveling wave model, numerical scheme, parallel algorithm, simulation, beam improvement.

AMS Subject Classification: 65M06; 65Y05; 65Z05.

\section{Introduction}

High power high brightness edge-emitting (EE) broad area semiconductor (BAS) lasers and optical amplifiers are compact devices playing a key role in different laser technologies. EE BAS lasers and amplifiers (see Fig. 1(a)) have a relatively simple geometry allowing an efficient pumping through a broad electric contact on the top of the device and are able to operate at the high power (tens of Watts) regimes. BAS devices, however, have one serious drawback: once operated at high power regimes, they suffer from a relatively low beam quality which is due to simultaneous irregular contribution of different lateral and longitudinal modes. As a result, the emitted optical beam has undesirable 
(a)

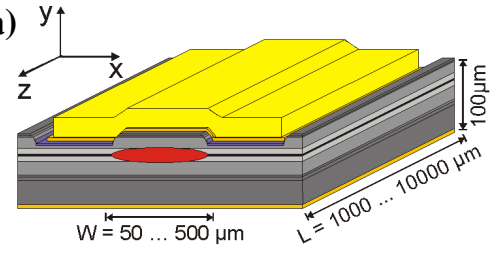

(c)

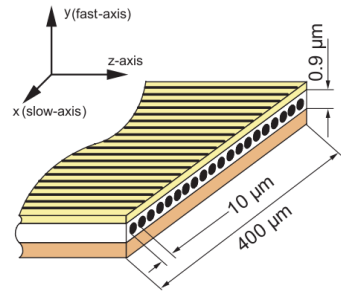

(d)

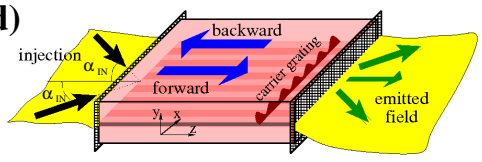

(b) Ridge waveguide DFB

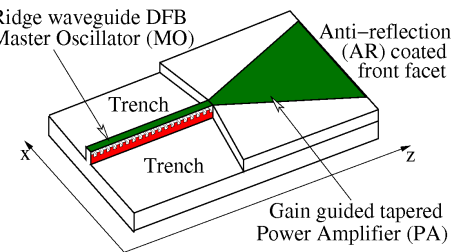

outcoupling branch

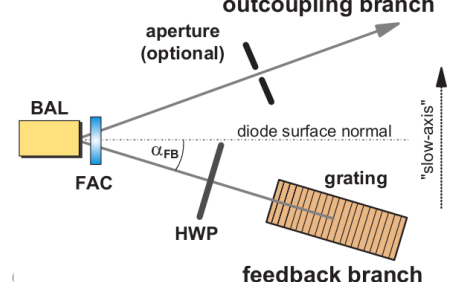

(e)

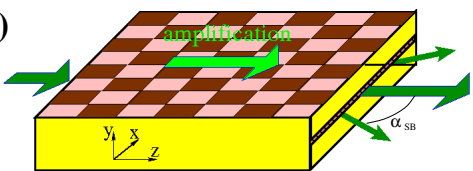

Figure 1. Schemes of a standard EE BAS device (a) and different modifications admitting a beam quality improvement. (b): master-oscillator tapered power-amplifier laser. (c): striped array BAS laser with an off-axis external cavity. (d): BAS laser with a dual angular plane wave injection. (e): BAS amplifier with a spatially periodic electrical contact.

broad optical and lateral spectra. A quality improvement of the beam amplified in BAS amplifiers or generated by BAS lasers is a very important issue of the modern semiconductor laser technology, and there are several BAS device concepts allowing some improvement of the optical beam.

Mathematical modeling, simulations and analysis play a significant role in optimization of existing devices or creation of novel design concepts. Typically, the length ( $z$ dimension) and width ( $x$-dimension) of EE BAS devices (see Fig. 1(a)) are in a few millimeter and hundreds of micrometer range, respectively, whereas the height ( $y$ dimension) of the active zone where the optical beam is generated and amplified is not larger than a micrometer. There exist different models describing stationary and/or dynamical states in EE BAS devices. The most complicated of them is resolving temporal-spatial dynamics of full semiconductor equations accounting for microscopic effects and is given by $(3+1)$-dimensional PDEs [4]. More simple, but still appropriate models for BAS devices can be derived assuming paraxial approximation of the Maxwell equations and averaging over the vertical $y$ direction. Several versions of the resulting dynamical (2+1)-dimensional Traveling-Wave (TW) model were simulated and analyzed by different authors in Refs. [2, 9, 15, 18, 29].

Since full 3-dimensional dynamical simulations of semiconductor devices with different spatial and temporal scales in a reasonable time are not possible, we are considering the $(2+1)$-dimensional TW model described briefly in this paper. This model is based on the TW equations for counter-propagating and laterally diffracted slowly varying optical fields which are coupled to the ODE 
for induced polarizations and diffusive rate equation for carrier densities [3,25]. The well-posedness of this model was studied in [14], while different algorithms used for the numerical integration of the model were investigated in $[5,6,13]$. A possibility to formulate various boundary conditions and compact numerical approximations of these conditions are considered in [21].

Precise dynamic simulations of long and broad or tapered devices and tuning/optimization of the model with respect to one or several parameters, require huge CPU time and memory resources. A proper resolution of rapidly oscillating fields in typical BAS devices on a sufficiently large optical frequency range requires a fine space $\left(10^{6}-10^{7}\right.$ mesh points) and time (up to $10^{6}$ points for typical 5 ns transients) discretization. Dynamic simulations of such devices can easily take one or even several days of computations on a single processor computer. Some speedup of computations can be achieved by using problemdependent relations of the grid steps, including also variable steps in the lateral dimension. All these grid optimizations, however, are not sufficient when oneor a few- parameter studies with the simulation times up to 1000 ns should be performed. It is obvious, that the required computations in an acceptable time can only be done by means of parallel computers and parallel solvers.

In this paper we present a split-step Fourier method based numerical algorithm for the integration of the $2+1$ dimensional traveling wave model of BAS devices. The approximation of all equations is done on the same space grid, while a staggered grid was used in our previous papers [5,6]. A parallel version of this finite difference scheme is developed. The method of domain decomposition is used to parallelize the sequential algorithm. The obtained parallel algorithm is implemented on the parallel cluster of computers at the Weierstrass Institute in Berlin. Results of computational experiments are presented and analyzed. In particular a good strong scaling of the presented parallel algorithm is obtained, e.g. a speed-up $S_{n} \approx 25$ is obtained on 32 processors for a fixed problem size. The tests of weak scaling also are done, the results of computational experiments show a monotonic increase of speed-up $S_{n}$ for larger problem sizes.

The proposed numerical algorithm was successfully used by one of the authors for simulations of different BAS lasers and amplifiers (see Fig. 1(b)-(e)) with an improved quality of the beam. The master-oscillator (MO) tapered power-amplifier (PA) laser shown in Fig. 1(b) was analyzed theoretically and experimentally in $[24,25,28]$. A narrow waveguide of the MO enables an improvement of the lateral profile of the beam, which later is amplified in the tapered PA part of the device. However, even a small optical feedback at the PA facet can easily excite multiple longitudinal modes which degrade the performance of the device. Fig. 1(c) represents the striped array BAS device with the off-axis external feedback [11]. When the angle of the external cavity is in a proper relation with the lateral period of the striped electrical contact, one can achieve an effective suppression of all but one lateral modes. The emitted field with an improved quality then should be collected at the angle adjoint to the external cavity. The basic drawbacks of this configuration are a large size and a moderate power of the collected field. Fig. 1(d) and (e) represent two theoretically proposed configurations of the BAS laser and amplifier, respectively. 
A pair of coherently injected plane waves at the adjoint angles to the laser axis (Fig. 1(d)) can create a periodic carrier grating, which in turn can suppress all but one lateral modes of the laser $[22,23]$. Finally, Fig. 1(e) represents a situation where a properly designed periodic modulation of the electrical contact or active zone in both spatial directions can lead to a significant improvement of the amplified beam in BAS amplifiers $[10,19]$. Simulations of all these examples are briefly presented at the end of this paper. Some preliminary results of this paper are presented in short note [20].

\section{Mathematical Model}

We use the traveling wave (TW) model [25] for simulations of BAS devices. It is a $2+1$-dimensional system of PDEs which governs the spatio-temporal dynamics of the complex slowly varying amplitudes of the counter-propagating fields $E^{ \pm}\left(z^{\prime}, x^{\prime}, t^{\prime}\right)$, polarization functions $P^{ \pm}\left(z^{\prime}, x^{\prime}, t^{\prime}\right)$ and real carrier density function $N\left(z^{\prime}, x^{\prime}, t^{\prime}\right)$. After rescaling of the coordinates

$$
z=\frac{1}{1 \mathrm{~mm}} z^{\prime}, \quad x=\sqrt{\frac{k_{0} \bar{n}}{1 \mathrm{~mm}}} x^{\prime}, \quad t=\frac{c_{0}}{n_{g} \cdot 1 \mathrm{~mm}} t^{\prime},
$$

$\left(\bar{n}\right.$ : background refraction index, $k_{0}=\frac{2 \pi}{\lambda_{0}}$ : central wavenumber, $\lambda_{0}$ : central wavelength, $c_{0}$ : speed of light in vacuum, $n_{g}$ : group velocity index) and a suitable normalization of parameters and dynamical variables, the TW model can be written as follows [5]:

$$
\begin{aligned}
& \left(\frac{\partial}{\partial t} \pm \frac{\partial}{\partial z}\right) E^{ \pm}=-\frac{i}{2} \frac{\partial^{2}}{\partial x^{2}} E^{ \pm}-i\left[\beta\left(N,\|E\|^{2}\right)-i \frac{\mathcal{D}}{2}\right] E^{ \pm}-i \kappa^{\mp} E^{\mp}+F_{s p}^{ \pm} \\
& \frac{\partial}{\partial t} P^{ \pm}=i \bar{\omega} P^{ \pm}+\bar{\gamma}\left(E^{ \pm}-P^{ \pm}\right) \\
& \frac{1}{\mu} \frac{\partial}{\partial t} N=D \frac{\partial^{2}}{\partial x^{2}} N+I(z, x)-R(N)-\Re e \sum_{\nu= \pm} E^{\nu *}\left[G\left(N,\left|E^{ \pm}\right|^{2}\right)-\mathcal{D}\right] E^{\nu}
\end{aligned}
$$

where $\|E\|^{2}=\left|E^{+}\right|^{2}+\left|E^{-}\right|^{2}$ is proportional to the local field intensity, the complex factors $\kappa^{+}$and $\kappa^{-}$represent the coupling of the counterpropagating fields by the (optional) integrated Bragg grating, and the propagation factor $\beta$ is defined by

$$
\beta\left(N,\left|E^{ \pm}\right|^{2}\right)=\Delta-\tilde{n}(N)+\frac{i\left(G\left(N,\|E\|^{2}\right)-\alpha\right)}{2} .
$$

The operator $\mathcal{D}$ and functions $R, G, \tilde{n}$ in the formulas above denote the Lorentzian approximation of the material gain dispersion, the spontaneous recombination of carriers, the gain peak value, and the refractive index change, respectively:

$$
\begin{gathered}
R(N)=A N+B N^{2}+C N^{3}, \quad \mathcal{D} E^{ \pm}=\bar{g}\left(E^{ \pm}-P^{ \pm}\right), \\
G\left(N,\|E\|^{2}\right)=\frac{g^{\prime} N_{t r}}{1+\varepsilon\|E\|^{2}} \log \left(\frac{\max \left(N, N_{*}\right)}{N_{t r}}\right), \quad \tilde{n}(N)=2 \sigma N_{t r} \sqrt{N / N_{t r}} .
\end{gathered}
$$


In general, this model should be considered in the unbounded region $Q=$ $Q_{z, x} \times(0, T]$, where $Q_{z, x}=\{(z, x):(z, x) \in(0, L) \times \mathbf{R}\}$ is the spatial domain, $L$ represents the length of the device, $x$ is the coordinate of the unbounded lateral axis of the device, and $T$ defines the length of the time interval where we perform the integration. In our numerical simulations we choose a large enough lateral interval $[-X, X]$ containing the considered BAS device and assume that the field and carrier density functions $E^{ \pm}$and $N$ are periodic along the lateral axis:

$$
E^{ \pm}(z, x+2 X, t)=E^{ \pm}(z, x, t), \quad N(z, x+2 X, t)=N(z, x, t), \quad(z, x, t) \in Q .
$$

This assumption restricts our considerations of the model equations to the truncated domain $Q^{X}=Q_{z, x}^{X} \times(0, T], Q_{z, x}^{X}=\{(z, x):(z, x) \in(0, L) \times[-X, X]\}$. We note, the challenges of the right selection and accurate numerical approximation of the appropriate boundary conditions (BCs) is well known, see [1]. In our previous paper [5] we have investigated the performance of the standard Crank-Nicolson scheme supplemented with different BCs, including the exact discrete transparent boundary conditions (DTBCs) [8], and the approximate DTBCs suggested by Szeftel [26]. High order numerical approximations of BCs on nonuniform space grids are considered in $[7,21]$.

The boundary conditions for the optical fields $E^{ \pm}$at the device facets $(z, x) \in 0 \times[-X, X]$ and $(z, x) \in L \times[-X, X]$ in $(2.1)$ are given by

$$
\begin{aligned}
& E^{+}(0, x, t)=r_{0}(x) E^{-}(0, x, t)+a(x, t), \\
& E^{-}(L, x, t)=r_{1}(x) E^{+}(L, x, t)+F\left[E^{+}(L, \cdot, t-\tau)\right](x, t),
\end{aligned}
$$

for $(x, t) \in[-X, X] \times[0, T]$, where $r_{0}$ and $r_{1}$ are the field amplitude reflectivity coefficients, $a(x, t)$ denotes the complex amplitude of the possible optical field injection, and the operator $F$ represents another possible optical source determined by the delayed optical field re-injected back into the device [11].

The initial conditions

$$
E^{ \pm}(z, x, 0)=E_{0}^{ \pm}(z, x), \quad P^{ \pm}(z, x, 0)=P_{0}^{ \pm}(z, x), \quad N(z, x, 0)=N_{0}(z, x)
$$

are defined for all $(z, x) \in Q_{z, x}^{X}$. If properly stated, they are not very important, since after some transients the simulated trajectories approach one of the existing stable attractors.

The coefficients $\Delta, \alpha, g^{\prime}, \sigma, N_{t r}, N_{*}, \varepsilon, \mu, D, I, A, B$ and $C$ represent the static detuning due to the built-in refractive index profile, the internal losses of the field, the differential gain, the differential index, the carrier density at the transparency, the gain clamping carrier density, the nonlinear gain compression, the scaling factor related to the ratio of the photon and carrier life times, the carrier diffusion coefficient, the current injection density, and three recombination factors, respectively. The parameters $\bar{g}, \bar{\omega}$ and $\bar{\gamma}$ define the Lorentzian fit of the gain profile and denote the amplitude, the central frequency and the half width at half maximum of this Lorentzian. Finally, the random function $F_{s p}^{ \pm}$represents the spontaneous emission.

Most of the parameters entering model equations are allowed to be spatially non-homogeneous and discontinuous depending on the device geometry. 
More details about the meaning and typical values of these parameters can be found in $[3,25]$. Normalization of the equations and typical values of the normalized parameters were given in [5]. It is noteworthy, that typical values of $\bar{\gamma} \approx 10^{2} \div 10^{3}$ and $\mu \approx 10^{-3}$ represent the fast relaxation of the polarization functions $P^{ \pm}$and the slow dynamics of the carrier density $N$, respectively. Typical size of the dimensionless domain is determined by $X \approx 5 \div 30$ and $L \approx 1 \div 10$, whereas $D \approx 0.5$ and most of other parameters are of order $\mathcal{O}(1)$.

\section{Numerical Scheme}

The computation domain $Q^{X}$ is discretized using a uniform in space and time grid $Q_{h}^{X}=Q_{h, z, x}^{X} \times \omega_{h, t}$, where $Q_{h, z, x}^{X}=\omega_{h, z} \times \omega_{h, x}$, and

$$
\begin{aligned}
\omega_{h, x} & =\left\{x_{j}: x_{j}=j h_{x}, j=-J / 2, \ldots, J / 2-1, h_{x}=2 X / J\right\}, \\
\omega_{h, z} & =\left\{z_{k}: z_{k}=k h, k=0, \ldots, K, h=L / K\right\} \\
\omega_{h, t} & =\left\{t_{m}: t_{m}=m h, m=0, \ldots, M, M=T / h\right\} .
\end{aligned}
$$

The temporal discretization step $h$ is equal to the spatial step in $z$-direction, what allows an accurate simulation of the optical field propagation along the characteristic lines $z \pm t=$ const. We note also, that $h$ is the maximal allowed time step: its further increasing violates Courant-Friedrichs-Lewy condition and, therefore, the stability of the numerical scheme would be not satisfied. We note that in this paper we are using the collocated grid, while in $[5,6]$ the approximation is done on staggered grids.

All spatially depending parameters $P(z, x)$, spatially and temporarily depending functions $F(x, t)$ and unknown functions $U(z, x, t)$ in Eqs. (2.1)-(2.4) are approximated by their grid analogs defined on $Q_{h, z, x}^{X}$ or $Q_{h}^{X}$ :

$$
P_{k, j}=P\left(z_{k}, x_{j}\right), \quad F_{j}^{m}=F\left(x_{j}, t_{m}\right), \quad U_{k, j}^{m} \approx U\left(z_{k}, x_{j}, t_{m}\right) .
$$

When constructing numerical schemes we exploit a discrete Fourier transform of complex and real laterally-periodic functions $U(z, x, t)$, where $U=E^{ \pm}$ or $U=N$. This approach is especially useful for the estimation and approximation of higher order in $x$ linear differential operators of laterally-periodic systems. Namely, we assume that a complex function $U_{j}(z, t):=U\left(z, x_{j}, t\right)$ (representing the complex fields $E^{+}$and $E^{-}$) on the uniform lateral mesh $\omega_{h, x}$ can be expressed as a linear combination of the orthonormal grid-functions $e^{i \pi \ell x_{j} / X}, \ell=-J / 2, \ldots, J / 2-1$ :

$$
U_{j}(z, t)=\left[\mathcal{F}^{-1}\left(\left.\widehat{U}_{\ell}(z, t)\right|_{\ell=-J / 2} ^{J / 2-1}\right)\right]_{j}:=\frac{1}{J} \sum_{\ell=-J / 2}^{J / 2-1} \widehat{U}_{\ell}(z, t) e^{i \pi \ell x_{j} / X}
$$

where the Fourier coefficients $\widehat{U}_{\ell}(z, t)$ are defined as

$$
\widehat{U}_{\ell}(z, t)=\left[\mathcal{F}\left(\left.U_{j}(z, t)\right|_{j=-J / 2} ^{J / 2-1}\right)\right]_{\ell}:=\sum_{j=-J / 2}^{J / 2-1} U_{j}(z, t) e^{-i \pi \ell x_{j} / X}
$$


An approximation of the second lateral derivative of the function $U$ at any grid point $x_{j}$ now can be written as follows:

$$
\frac{\partial^{2}}{\partial x^{2}} U\left(z, x_{j}, t\right) \approx \frac{1}{J} \sum_{\ell=-J / 2}^{J / 2-1}\left(-\frac{\pi^{2} \ell^{2}}{X^{2}}\right) \widehat{U}_{\ell}(z, t) e^{i \pi \ell x_{j} / X} .
$$

When the function $U$ represents the carrier density $N$ and is real, it can be expressed as a linear combination of the real orthogonal grid-functions $\cos \left(\pi \ell x_{j} / X\right), \ell=0, \ldots, J / 2$, and $\sin \left(\pi \ell x_{j} / X\right), \ell=1, \ldots, J / 2-1$. An equivalent complex expression of this liner combination still can be written as (3.1) with the complex Fourier coefficients (3.2) satisfying the relations $\widehat{N}_{-J / 2}=$ $\widehat{N}_{-J / 2}^{*}$ and $\widehat{N}_{\ell}=\widehat{N}_{-\ell}^{*}, \ell=0, \ldots, J / 2-1$.

Splitting scheme. The TW model (2.1)-(2.4) is integrated numerically using a splitting scheme, where the lateral field diffraction and carrier diffusion are resolved with the fast Fourier transform, and the remaining coupled hyperbolic system in (2.1) is integrated along the characteristics using finite differences.

Various splitting techniques are used to construct efficient integrators of the Schrödinger type semi-discrete problems $[16,17,27]$. Here we adapt a general splitting technique to approximate specific equations of the given system of nonlinear differential equations. We note that the accuracy of approximation can be increased if the symmetrical version of the splitting scheme would be used.

Let us assume, that the grid functions $E_{k, j}^{ \pm, m}, P_{k, j}^{ \pm, m}$ and $N_{k, j}^{m}$ are known for the time layer $t^{m}$. To find the values of these functions at the new time layer $t^{m+1}$ we proceed as follows. In the first step of our algorithm we make a simple prediction of the carrier density at the new time layer:

$$
\begin{aligned}
& \frac{\tilde{N}_{k, j}^{m+1}-N_{k, j}^{m}}{\mu h}=-\left(G\left(N_{k, j}^{m},\left\|E_{k, j}^{m}\right\|^{2}\right)-\bar{g}\right)\left\|E_{k, j}^{m}\right\|^{2}-\bar{g} \Re e \sum_{\nu= \pm} E_{k, j}^{\nu, m *} P_{k, j}^{\nu, m} \\
& +I_{k, j}-\frac{\tilde{N}_{k, j}^{m+1} R\left(N_{k, j}^{m}\right)}{N_{k, j}^{m}}, \quad k=0, \ldots, K, j=-J / 2, \ldots, J / 2-1 .
\end{aligned}
$$

Due to the slow carrier dynamics (the diffusion is moderate and the factor $\mu$ is small) we use a simple explicit scheme with a totally ignored carrier diffusion at this step.

In the next step we neglect the field diffraction and find intermediate approximations for the optical fields and new polarization functions:

$$
\begin{aligned}
& \frac{\widetilde{E}_{k, j}^{ \pm, m+1}-E_{k \mp 1, j}^{ \pm, m}}{h}=-\frac{\bar{g}_{k, j}\left(\widetilde{E}_{k, j}^{ \pm, m+1}-P_{k, j}^{ \pm, m+1}\right)+\bar{g}_{k \mp 1, j}\left(E_{k \mp 1, j}^{ \pm, m}-P_{k \mp 1, j}^{ \pm, m}\right)}{4} \\
& -\frac{i}{2}\left[\beta\left(\widetilde{N}_{k, j}^{m+1},\left\|E_{k, j}^{m}\right\|^{2}\right) \widetilde{E}_{k, j}^{ \pm, m+1}+\beta\left(N_{k \mp 1, j}^{m},\left\|E_{k \mp 1, j}^{m}\right\|^{2}\right) E_{k \mp 1, j}^{ \pm, m}\right] \\
& -i \frac{\kappa_{k, j}^{\mp} \widetilde{E}_{k, j}^{\mp, m+1}+\kappa_{k \mp 1, j}^{\mp} E_{k \mp 1, j}^{\mp, m}}{2}, \quad k, k \mp 1 \in\{0, \ldots, K\} ;
\end{aligned}
$$




$$
\begin{gathered}
\widetilde{E}_{0, j}^{+, m+1}=r_{0, j} \widetilde{E}_{0, j}^{-, m+1}+a_{j}^{m+1}, \quad \widetilde{E}_{K, j}^{-, m+1}=r_{1, j} \widetilde{E}_{K, j}^{+, m+1}+F_{h}\left[E_{K, \cdot}^{+, m+1-\frac{\tau}{h}}\right], \\
P_{k, j}^{ \pm, m+1}=\frac{\bar{\gamma}_{k, j}\left(1-e^{\left(i \bar{\omega}_{k, j}-\bar{\gamma}_{k, j}\right) h}\right)}{\bar{\gamma}_{k, j}-i \bar{\omega}_{k, j}} \widetilde{E}_{k, j}^{ \pm, m+1}+e^{\left(i \bar{\omega}_{k, j}-\bar{\gamma}_{k, j}\right) h} P_{k, j}^{ \pm, m} \\
k=0, \ldots, K, j=-J / 2, \ldots, J / 2-1 .
\end{gathered}
$$

Here the stiff ODE for the polarization functions $P^{ \pm}$in $(2.1)(\bar{\gamma}$ is large!) is resolved using an exponentially weighted scheme with the forward values for $E^{ \pm}$, which ensures, that in the limit $\bar{\gamma} \rightarrow \infty$ the discretized solutions $P^{ \pm}$ converge to $E^{ \pm}[25]$.

We note, that the scheme above is linear with respect to $\widetilde{E}_{k, j}^{ \pm, m+1}$ and $P_{k, j}^{ \pm, m+1}$ and can be separately resolved for each $k=0, \ldots, K$.

In the final step of our algorithm we take into account the carrier diffusion and field diffraction. Namely, we solve the linear equations

$$
\frac{\partial}{\partial t} N=\mu D \frac{\partial^{2}}{\partial x^{2}} N, \quad\left(\frac{\partial}{\partial t} \pm \frac{\partial}{\partial z}\right) E^{ \pm}=-\frac{i}{2} \frac{\partial^{2}}{\partial x^{2}} E^{ \pm}
$$

within the time (and space) interval of length $h$, whereas the initial conditions are given by the previously obtained estimates $\widetilde{N}$ and $\widetilde{E}^{ \pm}$. To integrate these equations we use a lateral discretization of the functions $N$ and $E^{ \pm}$, approximate their second lateral derivatives by (3.3) and solve the resulting systems of the differential equations in the (lateral) Fourier domain:

$$
\widehat{N}_{\ell}(z, t+h)=e^{-\mu D \frac{\pi^{2} \ell^{2}}{X^{2}} h} \widehat{N}_{\ell}(z, t), \quad \widehat{E}_{\ell}^{ \pm}(z \pm h, t+h)=e^{i \frac{\pi^{2} \ell^{2}}{2 X^{2}} h} \widehat{E}_{\ell}^{ \pm}(z, t)
$$

The inverse discrete Fourier transform (3.1) and the discretization of the functions $N$ and $E^{ \pm}$along the longitudinal $z$ direction give us the following equations, which complete the description of our numerical scheme:

$$
\begin{aligned}
N_{k, j}^{m+1} & =\frac{1}{J} \sum_{\ell=-J / 2}^{J / 2-1}\left[e^{-\mu D \frac{\pi^{2} \ell^{2}}{X^{2}} h} \sum_{s=-J / 2}^{J / 2-1} \widetilde{N}_{k, s}^{m+1} e^{-i \frac{2 \pi \ell s}{J}}\right] e^{i \frac{2 \pi \ell j}{J}}, \\
E_{k, j}^{ \pm, m+1} & =\frac{1}{J} \sum_{\ell=-J / 2}^{J / 2-1}\left[e^{i \frac{\pi^{2} \ell^{2}}{2 X^{2}} h} \sum_{s=-J / 2}^{J / 2-1} \widetilde{E}_{k, s}^{ \pm, m+1} e^{-i \frac{2 \pi \ell s}{J}}\right] e^{i \frac{2 \pi \ell j}{J}}, \\
k & =0, \ldots, K, j=1, \ldots, J .
\end{aligned}
$$

\section{Parallelization}

The numerical scheme (3.4)-(3.6) is well suited for the execution on parallel computers by using the domain decomposition technique. To distribute the computational work among different processes $\zeta_{l}, l=1, \ldots, n$ we decompose the computational grid $Q_{h}^{X}$ along the longitudinal $z$-direction into $n$ nonoverlapping sub-grids $Q_{h}^{X, l}$. In order to reach a load balancing, the first $(n-1)$ 


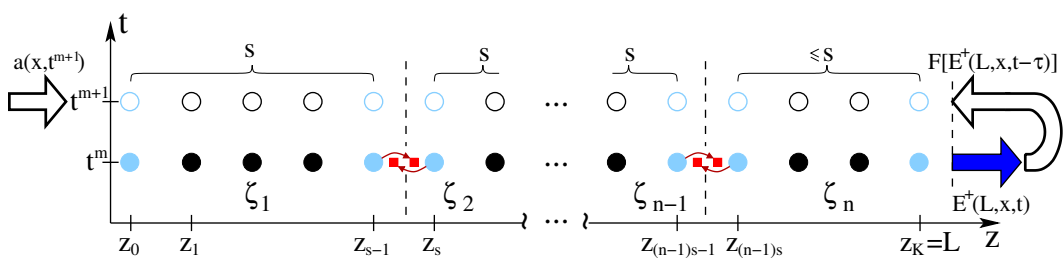

Figure 2. A schematic representation of the computational grid ( $z$ and $t$ coordinates only). Vertical dashed lines indicate splitting of the grid $Q_{h}^{X}$ to smaller sub-grids $Q_{h}^{X, l}$. Full and empty bullets represent the actual (already computed) and the next time layers, respectively. Arrows indicate data streams which should be read or recorded by different processes $\zeta_{l}$ before the next time iteration. Small boxes: ghost points of the sub-grid containing an information received from the corresponding border point (light blue bullets) of the adjacent sub-grid.

sub-grids $Q_{h}^{X, l}$ have $s$ longitudinal grid points $z_{k}$ each, whereas the corresponding grid point number in the last sub-grid is smaller or equal to $s$. In such a manner, any process $\zeta_{l}$ operates on the sub-grid

$$
\begin{aligned}
& Q_{h}^{X, l}=\omega_{h, z}^{l} \times \omega_{h, x} \times \omega_{h, t}, \quad \omega_{h, z}^{l}=\omega_{h, z} \cap[(l-1) s h, \min \{(l s-1) h, L\}], \\
& \quad s=\lceil(K+1) / n\rceil, l=1, \ldots, n .
\end{aligned}
$$

A schematic representation of the full computational grid and its splitting to smaller sub-grids is given in Fig. 2.

Before computing the grid functions at the next time layer (empty bullets in Fig. 2) each process $\zeta_{l}$ needs to exchange the current time layer (full bullets) values of $E^{ \pm}, P^{ \pm}$and $N$ at the boundaries of the sub-grid $Q_{h}^{X, l}$ (light blue bullets in the same figure) with the adjacent processes $\zeta_{l-1}$ and $\zeta_{l+1}$. This information is recorded to the specially created ghost grid points (small full boxes) at the adjacent side of the sub-grids $Q_{h}^{X, l-1}$ and $Q_{h}^{X, l+1}$. The left and right ghost points of the sub-grid $Q_{h}^{X, l}$ in the consequent computations of the process $\zeta_{l}$ are treated like standard grid points $\left(z_{(l-1) s-1}, x_{j}, t^{m}\right)$ and $\left(z_{l s}, x_{j}, t^{m}\right)$ which, in general, are out of the scope of the process $\zeta_{l}$. Here we are not considering data communication algorithms, when exchange of data is overlapped with computations in the region where the ghost points are not included into the stencil of the discrete scheme.

The processes $\zeta_{1}$ and $\zeta_{n}$ operating on the end sub-grids $Q_{h}^{X, 1}$ and $Q_{h}^{X, n}$ have no left or right adjacent sub-grid. The required sub-grid boundary information in these cases is given by the longitudinal boundary conditions (2.3) including optional optical injection and optical feedback functions $a(x, t)$ and $F\left[E^{+}(L, x, t-\tau)\right]$ (empty in-pointing arrows in the same figure).

In addition to updating the solution of the scheme (3.4)-(3.6) on the subgrid $Q_{h}^{X, n}$, the last process $\zeta_{n}$ records the emitted field $E^{+}(L, x, t)$ and calculates distributions of the optical feedback (if considered). Thus, the fact that $Q_{h}^{X, n}$ has, possibly, less grid points than the other sub-grids could be advantageous seeking to speed up the simulations. In order to preserve a load balance among processes it can be recommended to reduce the size of the last sub-grid even more, if the post processing tasks are even more time consuming. 


\subsection{Scalability analysis of the parallel algorithm}

In this section we present a detailed scalability analysis of the constructed parallel algorithm. It is easy to estimate the costs of the sequential scheme to compute a solution at one time step

$$
W=c_{1} K J \log J
$$

where the factor $J \log J$ is due to application of FFT.

Now let us estimate the complexity of the proposed parallel algorithm. The computation costs are equal to

$$
T_{n}^{1}=c_{1}\lceil K / n\rceil J \log J .
$$

Here we are not taking into account the fact that the constant $c_{1}$ in the estimation of complexity of the parallel algorithm can be smaller than for the sequential algorithm due to better cashing of smaller size parallel subproblems. Thus we are considering the worst case scenario.

Next we will estimate costs of communication among processors. The implementation of the given parallel algorithm requires only local send/receive communications of $c_{1} J$ data items between neighbor processes and each process has at most two neighbours. All processes $\zeta_{1}$ are divided into two groups, depending if index $l$ is an even or odd number. Then the well-known red-black parallel data communication protocol is applied. The communication costs can be estimated as [12]

$$
T_{n}^{2}=c_{2}(\alpha+\beta J)
$$

where $\alpha$ denotes the message startup time and $\beta$ is the time required to send one element of data.

Thus the total complexity of the parallel algorithm is equal to

$$
T_{n}=c_{1}\lceil K / n\rceil J \log J+c_{2}(\alpha+\beta J) .
$$

The scalability analysis of any parallel algorithm finds the rate at which the size of the sequential algorithm $W$ needs to grow up with respect to the number of processes $n$ in order to maintain fixed the theoretical efficiency of the parallel algorithm $E=W /\left(n T_{n}\right)$. For a given efficiency $E$ the iso-efficiency function $T_{0}=g(n, E)$ is defined by the implicit equation [12]:

$$
W=\frac{E}{1-E} H(n, W) .
$$

The total overhead of the proposed parallel algorithm is given by

$$
H(n, W):=n T_{n}-W=c_{1}(n\lceil K / n\rceil-K) J \log J+n c_{2}(\alpha+\beta J) .
$$

In order to simplify the scalability analysis, we assume that for large size problems the following asymptotical estimate is valid

$$
H(n, W)=n c_{2} \beta J
$$


After simple computations we get from (4.2) the following iso-efficiency function, expressed with respect to the number of grid points in $z$ coordinate:

$$
K=\frac{E}{1-E} \frac{c_{2} \beta}{c_{1}} \frac{n}{\log J} .
$$

Thus in order to maintain a fixed efficiency $E$ of the parallel algorithm for a fixed number of grid points $J$, it is sufficient to preserve the same number of grid $\omega_{h, z}^{l}$ points per process. Also, it follows from (4.3) that the increase of $J$ reduces the dependence of $K$ on the number of processes $n$.

\subsection{Results of computational experiments}

The numerical scheme (3.4)-(3.6) and the parallel numerical algorithm were implemented by using the HPMPI library and executed on HP Blade server with 48 2xXeon5430/2666 Quad-Core nodes. The nodes are interconnected via Infiniband 4xDDR (20 Gbit/s).

We have performed set of simulations of three different size BA semiconductor devices. The first one (Problem 1) is located within the computational domain consisting of 640 steps in longitudinal $z$-direction and 1000 steps in lateral $x$-direction, whereas 1665 time iteration steps correspond to $100 \mathrm{ps}$ long transients. The computational domain of the second case (Problem 2) consists of 320 steps in longitudinal $z$-direction, 1000 steps in lateral $x$-direction, and 3331 time steps (200 ps long transients) are computed. The computational domain of the third problem (Problem 3) has 300 steps in $z$-direction and 1000 steps in $x$-direction, whereas 3278 time iteration steps correspond to 200 ps long transients.

Obtained performance results are presented in Table 1. Here $n=n_{n} \times n_{c}$ processes are used in computations, where $n_{n}$ denotes the number of nodes and $n_{c}$ denotes the number of multi-cores per one node. $\tau_{n}$ represents a CPU time needed for parallel $n$-process calculations and $S_{n}=\tau_{1} / \tau_{n}$ is an estimated speed-up of these computations. We note, that for comparison of the efficiency of parallelization we present the CPU time needed for time iterations only, i.e., we have excluded the CPU time used by initial data reading and final data writing procedures.

The main conclusion from the given results is that a general trend of the efficiency of the parallel algorithm (strong scaling), which is predicted by theoretical scalability estimates, is confirmed quite well. Even for moderate sizes of the discrete Problems 1, 2 and 3 the CPU time of the parallel algorithm monotonically decreases when the number of nodes $n_{d}$ increases and the number of multi-cores per node is fixed. The second conclusion is that up to 4 multi-cores per node can be used very efficiently for this type of nodes.

\section{Simulations of BAS Devices}

As it was mentioned before, an optical field in typical conventional high power BAS lasers is composed of multiple longitudinal and lateral optical modes, an emission is irregular, it has broad and irregular optical and lateral (angular) 
Table 1. Results of numerical experiments (time $\tau_{n}$ and speed-up $S_{n}$ ) for different numbers of processes $n=n_{n} \times n_{c}$ and different problems: Problem1 is solved on grid $(K, J, M)=$ $(640,1000,1665)$, Problem 2 on grid $(320,1000,3331)$ and Problem 3 on grid $(300,1000,3278)$.

\begin{tabular}{|c|c|c|c|c|c|c|c|c|}
\hline \multirow[t]{2}{*}{$n$} & \multirow[t]{2}{*}{$n_{n}$} & \multirow[t]{2}{*}{$n_{c}$} & \multicolumn{2}{|c|}{ Problem 1} & \multicolumn{2}{|c|}{ Problem 2} & \multicolumn{2}{|c|}{ Problem 3} \\
\hline & & & time $(\mathrm{s})$ & speed-up & time $(\mathrm{s})$ & speed-up & time $(\mathrm{s})$ & speed-up \\
\hline 1 & 1 & 1 & 1259.23 & & 1010.83 & & 876.084 & \\
\hline 2 & 1 & 2 & 656.467 & 1.918 & 524.064 & 1.929 & 453.311 & 1.933 \\
\hline 2 & 2 & 1 & 646.451 & 1.948 & 523.941 & 1.929 & 456.635 & 1.919 \\
\hline 4 & 1 & 4 & 355.158 & 3.546 & 273.97 & 3.690 & 253.47 & 3.456 \\
\hline 4 & 2 & 2 & 336.772 & 3.739 & 272.005 & 3.716 & 242.963 & 3.606 \\
\hline 4 & 4 & 1 & 334.721 & 3.762 & 267.812 & 3.774 & 242.947 & 3.606 \\
\hline 8 & 1 & 8 & 235.706 & 5.342 & 189.609 & 5.331 & 163.154 & 5.370 \\
\hline 8 & 2 & 4 & 186.794 & 6.742 & 148.795 & 6.794 & 127.956 & 6.846 \\
\hline 8 & 4 & 2 & 172.233 & 7.311 & 143.167 & 7.061 & 122.799 & 7.134 \\
\hline 8 & 8 & 1 & 170.194 & 7.398 & 144.308 & 7.005 & 132.82 & 6.596 \\
\hline 16 & 2 & 8 & 121.406 & 10.373 & 107.547 & 9.398 & 91.7906 & 9.544 \\
\hline 16 & 4 & 4 & 97.2596 & 12.947 & 78.7957 & 12.829 & 68.7539 & 12.742 \\
\hline 16 & 8 & 2 & 92.7069 & 13.582 & 77.146 & 13.102 & 68.7514 & 12.742 \\
\hline 16 & 16 & 1 & 90.7107 & 13.882 & 75.8379 & 13.330 & 68.1719 & 12.851 \\
\hline 32 & 4 & 8 & 67.7683 & 18.582 & 66.9519 & 15.098 & 55.6138 & 15.754 \\
\hline 32 & 8 & 4 & 57.8495 & 21.766 & 49.0234 & 20.621 & 36.993 & 23.683 \\
\hline 32 & 16 & 2 & 52.7375 & 23.878 & 47.4928 & 21.283 & 36.4114 & 24.061 \\
\hline 32 & 32 & 1 & 50.4645 & 24.954 & 50.1533 & 20.154 & 39.2695 & 22.310 \\
\hline 64 & 8 & 8 & 45.5049 & 27.673 & 48.1642 & 20.986 & 35.734 & 24.518 \\
\hline 64 & 16 & 4 & 36.9002 & 34.125 & 35.8065 & 28.230 & 19.1805 & 45.677 \\
\hline 128 & 16 & 8 & 36.2105 & 34.778 & 41.4629 & 24.384 & 23.4727 & 37.325 \\
\hline
\end{tabular}

spectra [29]. A simulated example of such a beam is given in Fig. 3. Even though the power of this computed field is high, an area of its applications is rather restricted.

Mathematical modeling and fast and robust numerical simulations are a powerful method used in optimization of the existing BAS devices or in creation of the novel design concepts for different real world applications. Below in this section we simulate several BAS devices (shown also in Fig. 1(b-e)) admitting an improved quality of the emitted beam. Note, that simulations of the effects discused in this section we typically performed using $n=16$ or $n=32$ processes on $n_{n}=4$ or $n_{n}=8$ nodes. The size of considered discrete problems is indicated within the captions of corresponding figures.

\subsection{Beam generation and amplification in MOPA lasers}

In general, an ideal MOPA laser (see Fig. 1(b)) should be able to maintain a good quality of the emitted beam. The basic idea of a perfect MOPA is as follows. The narrow MO part of the device generates a stable stationary optical field determined by a single optical mode. The high power emission is achieved during the propagation of this beam along the ideal non-reflective tapered PA 

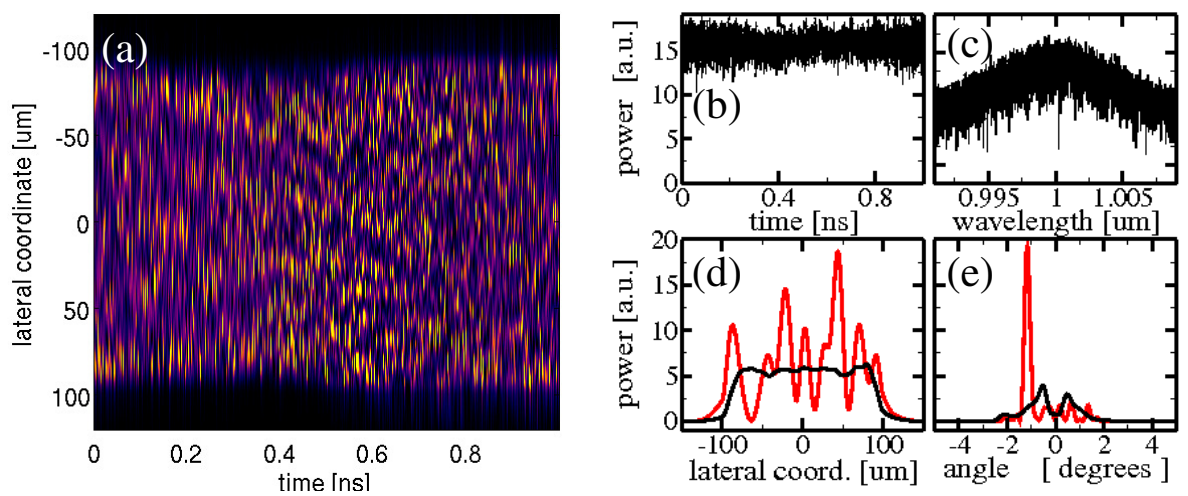

Figure 3. Simulated irregular dynamics of a conventional high power BAS laser. (a):

history of the near field intensities. (b): time trace of the emitted field intensity. (c): optical spectrum. (d): near fields. (e): far-fields. Black and red curves in panels (d) and (e) represent the time-averaged fields and the fields at some time instant. The discrete problem size is defined by $(K, J)=(320,1000)$ (the same as Problem 2 in Table 1$)$, whereas

$M \approx 1.67 \cdot 10^{4}$ corresponds to the represented $1 \mathrm{~ns}$ transient. $\tau_{16} \approx 7$ minutes.
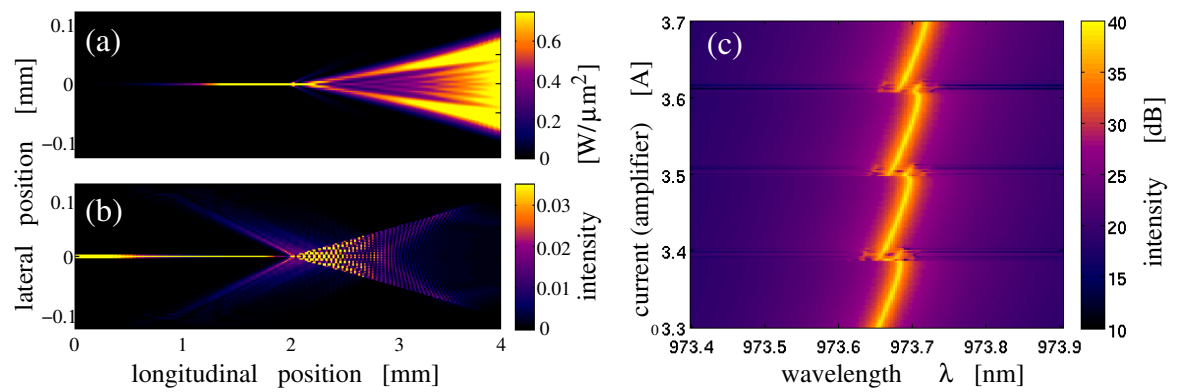

Figure 4. Simulations of the MOPA laser. (a) and (b): instantaneous distributions of the forward and backward field intensities $\left|E^{+}(z, x, t)\right|^{2}$ and $\left|E^{-}(z, x, t)\right|^{2}$. (c): dependence of the emitted field optical spectrum on the injected current in the power amplifier. The discrete problem size is defined by $(K, J)=(800,1000)$, whereas $M \approx 1.67 \cdot 10^{7}$ is needed for simulations of $1000 \mathrm{~ns}$ transients used for mapping plot (c). $\tau_{32} \approx 1$ week.

part of the device. The realistic MOPA devices, however, are not ideal. The perfect operation of the device is spoiled by the amplification of the random spontaneous emission $F_{s p}$ in the PA, by the small separation of the MO and PA electrical contacts, as well as by the non-vanishing field reflectivity (factor $r_{1}$ in Eq. (2.3)) at the PA facet of the device [25].

Panels (a) and (b) of Fig. 4 show the simulated intensity distributions of the forward- and backward-propagating fields of the MOPA laser operating at the required stable stationary regime. The generation of the stationary beam within the MO and its consequent amplification within the PA parts of the device can be well seen in Fig. 4 (a). Panel (b), however, represents all the unwanted effects mentioned above. Namely, the presence of the nonvanishing back-propagating field within the tapered PA is due to amplification 
of the spontaneous emission and, more important, due to residual field backreflection at the facet $\left(r_{1}=0.04\right.$ in this case). The small gap between the electrical contacts of the MO and PA at $z \approx 2 \mathrm{~mm}$ implies also some side-band scattering of the field.

For the present set of parameters the presence of the back-propagating field does no harm to the stationary operation of the laser. This situation, however, can change when tuning the injected current in the MO or PA parts of the device. The tuning of the injection implies the changes of the phase relations between complex forward- and back-propagating fields at the interface of the MO and PA what, in turn, can result unwanted bifurcations [6] leading to transitions between the states determined by different longitudinal modes. A typical simulated example of the transitions between different states is shown in Fig. 4 (c). For more details and comparisons with the experiments see [24,25, 28].

\subsection{Stripe-array lasers with an angular off-axis feedback}

Another example of an experimentally available BAS device admitting an improvement of the emitted beam quality is the BAS laser with the laterally $d_{x}$-periodic striped electrical contact and an optical feedback from the external mirror or grating located at the angle $\alpha_{F B}$ to the optical axis on the $(x, z)$ plane of the device: see Fig. 1(c). It was shown experimentally and numerically [11], that a proper choice of the feedback angle and the lateral period allows to get a high-quality emission at two opposite angles $\pm \alpha_{F B}$ (see Fig. 5(a), (b)).

For small $\alpha_{F B}$ and a small enough ratio of the laser width and the external cavity length in the non-scaled model, the Fresnel operator $F$ entering the boundary conditions (2.3) can be defined as [11]

$$
\begin{aligned}
& F\left[E^{+}\left(L, \cdot, t-2 d_{e c} / c_{0}\right)\right](x, t) \approx \nu e^{-i k_{0} \alpha_{F B} x} \mathcal{F} \bar{E}\left(t-2 d_{e c} / c_{0}\right), \\
& \nu=\left(1-\left|r_{1}\right|^{2}\right) \sqrt{\frac{-i}{2 d_{e c} \lambda_{0}}} e^{-i k_{0} 2 d_{e c}}, \quad \bar{E}(t)=\int_{x^{\prime} \in \mathbf{R}} E^{+}\left(L, x^{\prime}, t\right) e^{-i k_{0} \alpha_{F B} x^{\prime}} d x^{\prime},
\end{aligned}
$$

where $d_{e c}$ is the distance from the center of the right facet of the BAS diode to the external reflector, $2 d_{e c}+\alpha_{F B}\left(x+x^{\prime}\right)$ approximates the shortest distance between two lateral points $x^{\prime}$ and $x$ at the diode facet which the light takes to travel via the (infinitely broad) external reflector, whereas an operator $\mathcal{F}$ accounts for the spectral filtering by the external grating.

Assuming the stationary emission of the BAS device, one can easily show that the phase of the the complex angular feedback term changes linearly with a lateral coordinate $x$, so that each $\left(\lambda_{0} / \alpha_{F B}\right)$-shift of $x$ implies a change of this phase by factor $2 \pi$ (see grey dots in Fig. $5(\mathrm{e})$ and (f)). Thus, such an angular feedback can help enhancing a single lateral optical mode with the complex phases alternating periodically in $x$-direction (black dots in the same diagrams). The lateral periodicity of the electrical contact determines multiple equally separated field intensity peaks in each lateral cross-section of the laser: see black curve in Fig. 5(c). The best (most stable) emission can be expected for $d_{x} \approx \lambda_{0} / 2 \alpha_{F B}$, which supports the opposite phases of the complex fields at any two laterally-adjacent intensity maxima positions (black dots in Fig. 5(e)). The performed simulations could also predict a stable operation of the BAS 

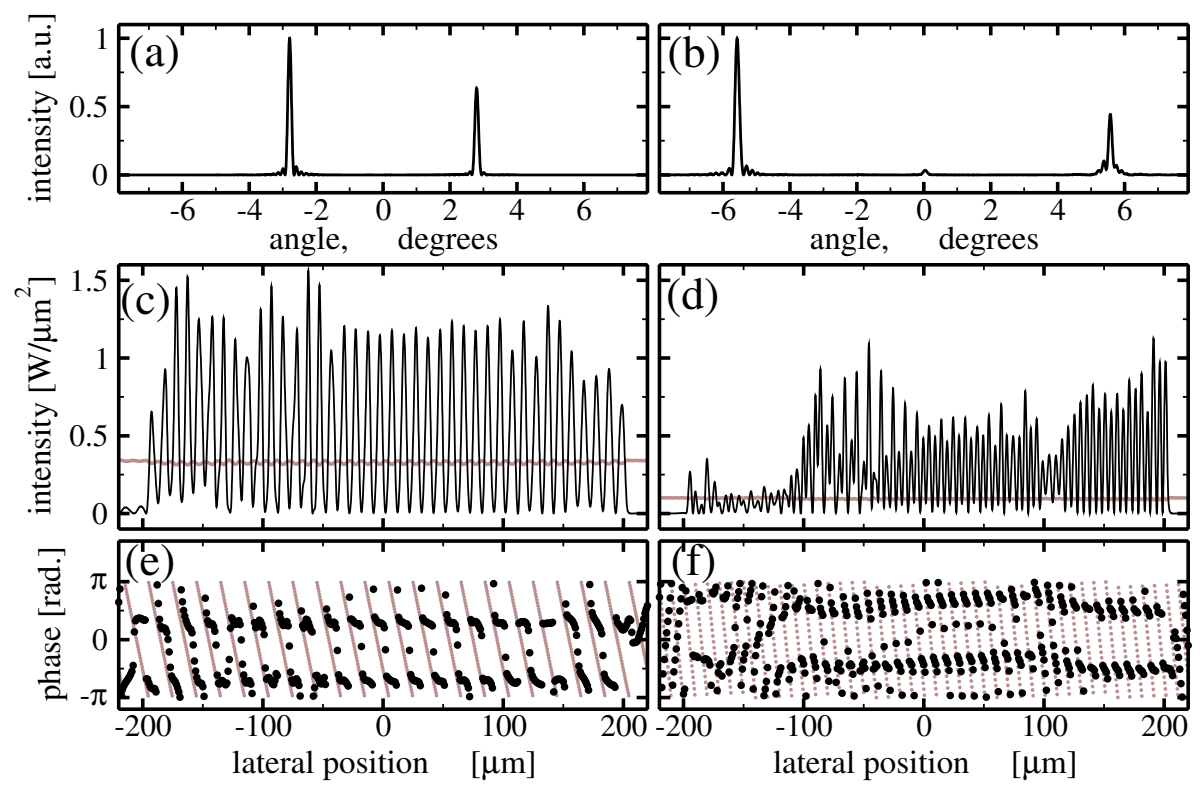

Figure 5. Simulations of BAS laser with a $d_{x}$-periodic striped electrical contact and an off-axis optical feedback located at $\alpha_{F B}=\lambda_{0} /\left(2 d_{x}\right) \approx 2.8^{\circ}$ [left panels (a), (c) and (e)] and $\alpha_{F B}=\lambda_{0} / d_{x} \approx 5.6^{\circ}$ [right panels (b), (d) and (f)]. (a) and (b): far fields. (c) and (d): near field intensities. (e) and (f): near field phases. Grey lines and bullets in (c,d,e,f) represent intensity and phase of the field re-injected into the BAS laser. The discrete problem size is defined by $(K, J)=(300,1000)$ (the same as Problem 3 in Table 1), whereas $M \approx 1.66 \times 10^{5}$ is needed for simulations of the required 10 ns transients. $\tau_{16} \approx 1$ hour.

laser with doubled feedback angle, $\alpha_{F B}=\lambda_{0} / d_{x}$ (see Fig. 5(d) and (f)), which later was confirmed experimentally [11].

\subsection{Stabilization of BAS lasers by a dual off-axis optical injection}

In our theoretical papers $[22,23]$ a new control method of BAS lasers was proposed, which, as we believe, should suppress all but one optical mode, i.e., should induce a stabilization of the emitted beam. This control is achieved by a pair of coherent optical plane waves injected into the BAS laser at the adjacent angles to the laser axis (see Fig. 1(d)). In the non-scaled model this optical injection is described by the function

$$
a(x, t)=a_{0} e^{i\left(\omega t+x k_{0} \alpha_{I N}-\pi / 2\right)}+a_{0} e^{i\left(\omega t-x k_{0} \alpha_{I N}+\pi / 2\right)}=2 a_{0} e^{i \omega t} \sin \left(\alpha_{I N} k_{0} x\right)
$$

entering boundary conditions (2.3). The parameters $\pm \alpha_{I N}$ and $\omega$ in the expression above denote the free space angles of the injected beams (see Fig. 1(d)) and the frequency detuning of the optical injection from the central frequency $k_{0} c_{0}$. The factor $\left|a_{0}\right|^{2}$ is proportional to the intensity of the optical field injected into the laser.

We have performed a series of simulations for the fixed detuning $\omega$ and increased intensity of the optical injection (i.e., parameter $\left|a_{0}\right|^{2}$ ). Some char- 


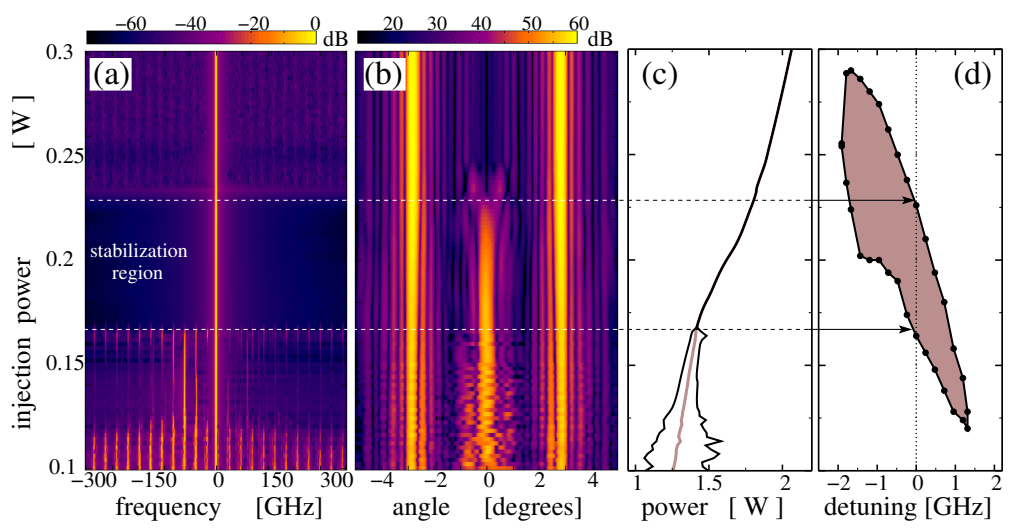

Figure 6. Stabilization of the BAS laser by the optical injection. (a): mapping of the optical spectra, (b): mapping of the far-fields computed at some time instant, (c): maximal, minimal and mean power of the emitted field for the increased injection power and fixed $\omega=0$. (d): laser stabilization region in injection power / frequency detuning $\omega$ plane. The discrete problem size is defined by $(K, J)=(300,1000)$ (the same as Problem 3 in Table 1 ), whereas $M \approx 8.3 \cdot 10^{6}$ is needed for simulations of 500 ns transients used for the 1-parameter representations (a), (b) and (c). $\tau_{16} \approx 2$ days. 20 such processes were required for the 2-parameter representation $(\mathrm{d})$.

acteristics (optical spectra, far-fields, field intensities) of typical observed dynamical states for $\omega=0$ and different injection intensities is summarized in Fig. 6. Here one can distinguish three qualitatively different regimes, separated by thin horizontal lines in Fig. 6. Once the injection intensity is too small, the spatial-temporal dynamics of the system is similar to that one of the free-running BAS laser. This can be recognized by multiple peaks of the optical spectrum (panel (a)), by scattered far-field instants (panel (b)), as well as by a non-stationary output field (differing minimal and maximal intensities in panel (c)). For moderate and large injected field intensities the laser operates at a continuous wave regime (a single spectral line in panel (a) and coinciding minimal and maximal powers in panel (c)). An inspection of the far-fields at these injections, however, allows us to distinguish two different regimes. Namely, for moderate injections we have a stationary state which has a well pronounced central angular component (a stabilized mode of the laser), whereas for larger injections only the angular components corresponding to the injected beam angles $\alpha_{I N}$ are present. In this regime our BAS laser is operating like an amplifier for the injected beams, but does not generate light by itself. Finally, panel (d) of the same figure, which shows a laser stabilization region in injection power/frequency detuning plane, summarizes a series of previously discussed simulations for different values of $\omega$.

\subsection{BAS amplifiers with periodically modulated electrical contacts}

An elegant way to improve the lateral beam profile in EE BAS amplifiers was suggested in the recent theoretical works $[10,19]$. It was shown, that a periodic 


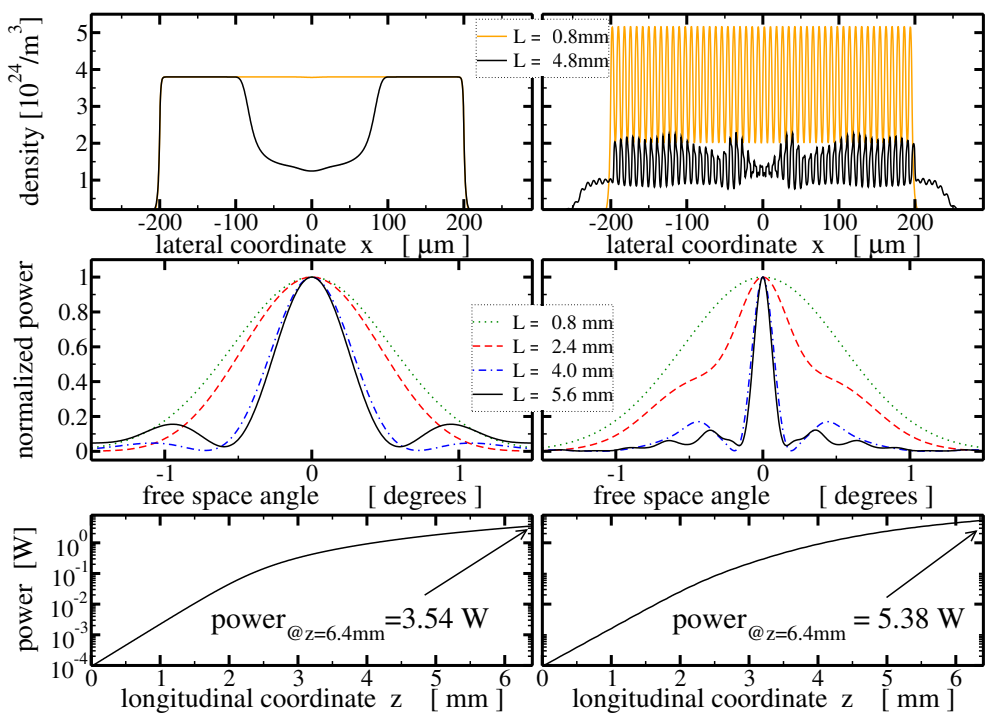

Figure 7. Amplification of the optical beam in $6.4 \mathrm{~mm}$ long EE BAS amplifiers with the conventional uniform electrical contact (left) and the periodically modulated contact with $d_{x}=8 \mu \mathrm{m}, d_{z}=400 \mu \mathrm{m}$, and $\mathcal{Q}=1.02$ (right). A total injected current in both cases is the same. First row: lateral distributions of the carrier density at $z=0.8 \mathrm{~mm}$ and $z=4.8 \mathrm{~mm}$. Second row: intensity of the far-fields computed for different longitudinal positions within the central $\pm 3^{\circ}$ segment. Third row: central part of the far-fields computed for $z=0.8,2.4$,

4 , and $5.6 \mathrm{~mm}$. The discrete problem size for computed $1 \mathrm{~ns}$ transient is defined by $(K, J, M)=(1280,2400,16666) . \tau_{16} \approx 1$ hour.

modulation of the gain and refractive index in both longitudinal and lateral directions (see Fig. 1(e)) can lead to a significant compression of the far-fields, what is desirable in the real world applications.

A crucial condition for the desired beam shaping is a proper choice of the lateral and longitudinal modulation periods $d_{x}$ and $d_{z}$, which in the nonnormalized model should satisfy the relation $\mathcal{Q}=2 d_{x}^{2} \bar{n} /\left(\lambda_{0} d_{z}\right) \approx 1$. In the present work we have performed simulations of the standard BAS amplifier (left panels of Fig. 7) and of the BAS amplifier with the periodically modulated electrical contact (right panels of the same figure) operating in moderate and high power regimes. In these regimes the carrier distribution is strongly depleted, causing also lateral irregularities in the carrier (i.e., gain and refractive index) modulation amplitudes: see the black curves in the first row panels of Fig. 7, representing the carrier densities at $z=4.8 \mathrm{~mm}$. The simulations have shown, that the proposed periodic modulation of the electrical contact implies the desired beam shaping. It can be seen when comparing the far-fields of the simple (left) and modulated amplifiers (right) at the middle row panels of this figure. It is noteworthy, that even though a part of the field amplified in the modulated device is radiated at the side band components at the angles $\pm \alpha_{S B}, \alpha_{S B} \approx \lambda_{0} / d_{x} \approx 7.2^{\circ}$, the intensity of the remaining central angle field still can be higher than that one of the field amplified in the conventional BAS device (compare the lower row panels). 


\section{Conclusions}

In conclusion, we have presented a numerical scheme for the $(2+1)$-dimensional PDE model describing the dynamics of BAS devices, and discussed its implementation on the parallel cluster of computers. The mathematical model and the numerical scheme were applied for the study of the beam stabilization in different configurations of BAS devices.

\section{Acknowledgments}

The authors would like to thank the referees for their comments which are helpful and constructive.

The work of M. Radziunas was supported by DFG Research Center MATHEON "Mathematics for key technologies: Modelling, simulation and optimization of the real world processes" and by EU FP7 ITN PROPHET, grant No. 264687. The work of R. Ciegis was supported by Eureka project E! 6799 POWEROPT "Mathematical modelling and optimization of electrical power cables for an improvement of their design rules".

\section{References}

[1] X. Antoine, A. Arnold, Ch. Besse, M. Ehrhardt and A. Schädle. A review of transparent and artificial boundary conditions techniques for linear and nonlinear Schrödinger equations. Commun. Comput. Phys., 4(4):729-796, 2008.

[2] S. Balsamo, F. Sartori and I. Montrosset. Dynamic beam propagation method for flared semiconductor power amplifiers. IEEE J. Select. Topics Quantum Electron., 2(2):378-384, 1996. http://dx.doi.org/10.1109/2944.577398.

[3] U. Bandelow, M. Radziunas, J. Sieber and M. Wolfrum. Impact of gain dispersion on the spatio-temporal dynamics of multisection lasers. IEEE J. Quantum Electron., 37(2):183-188, 2001. http://dx.doi.org/10.1109/3.903067.

[4] K. Böhringer and O. Hess. A full-time-domain approach to spatio-temporal dynamics of semiconductor lasers. I. Theoretical formulation. Progress Quantum Electron., 32(5-6):159-246, 2008.

http://dx.doi.org/10.1016/j.pquantelec.2008.10.002.

[5] R. Čiegis and M. Radziunas. Effective numerical integration of traveling wave model for edge-emitting broad-area semiconductor lasers and amplifiers. Math. Model. Anal., 15(4):409-430, 2010. http://dx.doi.org/10.3846/1392-6292.2010.15.409-430.

[6] R. Čiegis, M. Radziunas and M. Lichtner. Numerical algorithms for simulation of multisection lasers by using traveling wave model. Math. Model. Anal., 13(3):327-348, 2008. http://dx.doi.org/10.3846/1392-6292.2008.13.327-348.

[7] B. Ducomet, A. Zlotnik and I. Zlotnik. On a family of finite-difference schemes with approximate transparent boundary conditions for a generalized 1D Schrödinger equation. Kinetic and Related Models, 2(1):151-179, 2009. http://dx.doi.org/10.3934/krm.2009.2.151.

[8] M. Ehrhardt and A. Arnold. Discrete transparent boundary conditions for the Schrödinger equation. Riv. Mat. Univ. Parma, 6/4:57-108, 2001. 
[9] I. Fischer, O. Hess, W. Elsässer and E. Göbel. Complex spatio-temporal dynamics in the near-field of a broad-area semiconductor laser. Europhys. Lett., 35(8):579-584, 1996. http://dx.doi.org/10.1209/epl/i1996-00154-7.

[10] R. Herrero, M. Botey, M. Radziunas and K. Staliunas. Beam shaping in spatially modulated broad area semiconductor amplifiers. Optics Lett., 37(24):5233-5255, 2012. http://dx.doi.org/10.1364/OL.37.005253.

[11] A. Jechow, M. Lichtner, R. Menzel, M. Radziunas, D. Skoczowsky and A. Vladimirov. Stripe-array diode-laser in an off-axis external cavity: Theory and experiment. Optics Express, 17(22):19599-19604, 2009. http://dx.doi.org/10.1364/OE.17.019599.

[12] V. Kumar, A. Grama, A. Gupta and G. Karypis. Introduction to Parallel Computing: Design and Analysis of Algorithms. Benjamin/Cummings, Redwood City, 1994.

[13] I. Laukaitytè, R. Čiegis, M. Lichtner and M. Radziunas. Parallel numerical algorithm for the traveling wave model. In R. Čiegis, D. Henty, B. Kagstrom and J. Žilinskas(Eds.), Parallel Scientific Computing and Optimization, volume 27, pp. 237-251. Springer, 2009.

[14] M. Lichtner, M. Radziunas and L. Recke. Well posedness, smooth dependence and center manifold reduction for a semilinear hyperbolic system from laser dynamics. Math. Meth. Appl. Sci., 30(8):931-960, 2007. http://dx.doi.org/10.1002/mma.816.

[15] J.J. Lim, S. Sujecki, L. Lang, Z. Zhang, D. Paboeuf, G. Pauliat, G. LucasLeclin, P. Georges, R. MacKenzie, P. Bream, S. Bull, K.-H. Hasler, B. Sumpf, H. Wenzel, G. Erbert, B. Thestrup, P.M. Petersen, N. Michel, M. Krakowski and E.C. Larkins. Design and simulation of next-generation high-power, highbrightness laser diodes. IEEE J. Select. Topics Quantum Electron., 15(3):9931008, 2009. http://dx.doi.org/10.1109/JSTQE.2008.2011286.

[16] C. Lubich. On splitting methods for Schrödinger-Poisson and cubic nonlinear Schrödinger equations. Math. Comput., 77(264):2141-2153, 2008. http://dx.doi.org/10.1090/S0025-5718-08-02101-7.

[17] Ch. Neuhauser and M. Thalhammer. On the convergence of splitting methods for linear evolutionary Schrödinger equations involving an unbounded potential. BIT Numer. Math., 49(1):199-215, 2009. http://dx.doi.org/10.1007/s10543-009-0215-2.

[18] A. Perez Serrano, J. Javaloyes and S. Balle. Spectral delay algebraic equation approach to broad area laser diodes. IEEE J. Select. Topics Quantum Electron., 19(5):1502808, 2013. http://dx.doi.org/10.1109/JSTQE.2013.2246772.

[19] M. Radziunas, M. Botey, R. Herrero and K. Staliunas. Intrinsic beam shaping mechanism in spatially modulated broad area semiconductor amplifiers. Appl. Phys. Lett., 103(13):132101, 2013. http://dx.doi.org/10.1063/1.4821251.

[20] M. Radziunas and R. Čiegis. Modeling and simulations of beam stabilization in edge-emitting broad area semiconductor devices. In R. Wyrzykowski et al.(Ed.), Parallel Processing and Applied Mathematics (2), volume 8385 of Lecture Notes Comput. Sci., pp. 332-342. Springer, 2014.

[21] M. Radziunas, R. Čiegis and A. Mirinavičius. On compact high order finite difference schemes for linear Schrödinger problem on non-uniform meshes. Int. J. Numer. Anal. Model., 11(2):303-314, 2014. 
[22] M. Radziunas and K. Staliunas. Spatial rocking in broad area semiconductor lasers. Europhys. Lett., 95(1):14002, 2011. http://dx.doi.org/10.1209/0295-5075/95/14002.

[23] M. Radziunas and K. Staliunas. Spatial "rocking" for improving the spatial quality of the beam of broad area semiconductor lasers. In Proceedings of SPIE, volume 8432, p. 84320Q, 2012. http://dx.doi.org/10.1117/12.921544.

[24] M. Radziunas, V.Tronciu, U. Bandelow, M. Lichtner, M. Spreemann and H. Wenzel. Mode transitions in distributed-feedback tapered master-oscillator poweramplifier. Opt. Quantum Electron., 40(14-15):1103-1109, 2008. http://dx.doi.org/10.1007/s11082-009-9308-z.

[25] M. Spreemann, M. Lichtner, M. Radziunas, U. Bandelow and H. Wenzel. Measurement and simulation of distributed-feedback tapered master-oscillators power-amplifiers. IEEE J. Quantum Electron., 45(6):609-616, 2009. http://dx.doi.org/10.1109/JQE.2009.2013115.

[26] J. Szeftel. Design of absorbing boundary conditions for Schrödinger equation in $R^{d}$. SIAM J. Numer. Anal., 42(4):1527-1551, 2004. http://dx.doi.org/10.1137/S0036142902418345.

[27] M. Thalhammer, M. Caliari and Ch. Neuhauser. High-order time-splitting Hermite and Fourier spectral methods. J. Comput. Phys., 228(4):822-832, 2009. http://dx.doi.org/10.1016/j.jcp.2008.10.008.

[28] V.Tronciu, M. Lichtner, M. Radziunas, U. Bandelow and H. Wenzel. Improving the stability of distributed-feedback tapered master-oscillator power-amplifiers. Opt. Quantum Electron., 41(7):531-537, 2009. http://dx.doi.org/10.1007/s11082-009-9354-6.

[29] H. Wenzel. Basic aspects of high-power semiconductor laser simulation. IEEE J. Select. Topics Quantum Electron., 19(2):1-13, 2013. http://dx.doi.org/10.1109/JSTQE.2013.2246774. 